\title{
NOUVELLe
}

\section{Émergence \\ des cancers basocellulaires}

\section{Influence déterminante des fibroblastes du derme?}

Alexandre Valin, Marie-Françoise Avril, Thierry Magnaldo

\author{
A. Valin : FRE 2939 CNRS Génomes et cancers, \\ Institut Gustave Roussy, \\ 39 , rue Camille Desmoulins, \\ 94805, Villejuif Cedex 05, France; \\ FRE 3086 CNRS, Instabilités génétiques, \\ maladies rares et cancer, faculté de médecine \\ 28, avenue de Valombrose, \\ 06102 Nice Cedex, France. \\ M.F. Avril : AP-HP, Hôpital Cochin, Université Paris 5, \\ 75006 Paris, France. \\ T. Magnaldo : FRE 3086 CNRS, Instabilités génétiques, \\ maladies rares et cancer, faculté de médecine \\ 28, avenue de Valombrose, \\ 06102 Nice Cedex, France. \\ tmagnaldo@unice.fr
}

dans la croissance des

cellules tumorales et leur

caractère envahissant.

\section{Le syndrome de Gorlin}

Le syndrome de Gorlin, ou nævomatose basocellulaire, est une pathologie génétique rare (environ 1/60 000 naissances) associée à une forte prédisposition au $\mathrm{CBC}$ et, chez environ $5 \%$ des patients, au développement d'un médulloblastome. La maladie est transmise sur le mode autosomique et dominant. Elle peut être associée à des anomalies variables du développement (par exemple: côtes bifides ou surnuméraires, polysyndactylies). La survenue du premier CBC peut être observée dès l'adolescence chez le patient atteint de ce syndrome alors qu'elle apparaît en moyenne entre 60 et 65 ans dans la population générale. Ainsi, certains patients atteints de syndrome de Gorlin ont-ils, à 40 ans, souffert de plus d'une centaine de tumeurs. Le CBC n'est généralement pas une tumeur métastatique, mais sa multiplicité chez ces patients et parfois son agressivité locale posent des problèmes sérieux de prise en charge [4]. De façon très surprenante, environ $40 \%$ des $C B C$ développés par ces patients sont observés dans les zones cutanées couvertes [5], c'est-à-dire très peu ou pas exposées aux UV. Contrairement aux $\mathrm{CBC}$ sporadiques, le rayonnement UV ne constitue donc pas le facteur étiologi- que essentiel du CBC chez les patients porteurs d'un syndrome de Gorlin.

Bases moléculaires du syndrome de Gorlin : mutations du gène PATCHED Les bases moléculaires du syndrome de Gorlin sont connues depuis 1996 avec l'identification de mutations germinales dans le gène PATCHED associées à la maladie $[6,7]$. PATCHED code pour le récepteur de la protéine diffusible morphogène Sonic Hedgehog ( $\mathrm{SHH}$ ), orthologue humain de Hedgehog ( $\mathrm{Hh}$ ) identifiée chez la drosophile, et qui est essentielle à la polarisation des segments au cours du développement embryonnaire. En l'absence de son ligand, PATCHED réprime l'activation de la voie de signalisation en inhibant une protéine transmembranaire appelée SMOOTHENED (SMO). Dans les cellules cibles, la fixation de SHH à son récepteur libère l'activation de la voie dépendante de SMO et conduit à la transcription de gènes dont les produits sont impliqués dans le contrôle de l'équilibre entre différenciation et prolifération (notamment le facteur de transcription GLII et PATCHED) (Figure 1). Les nombreux mutants de drosophile et les pathologies humaines rares dus à la perte d'intégrité de la voie SHH soulignent son implication essentielle dans le contrôle des interactions tissulaires. Avec le syn- 


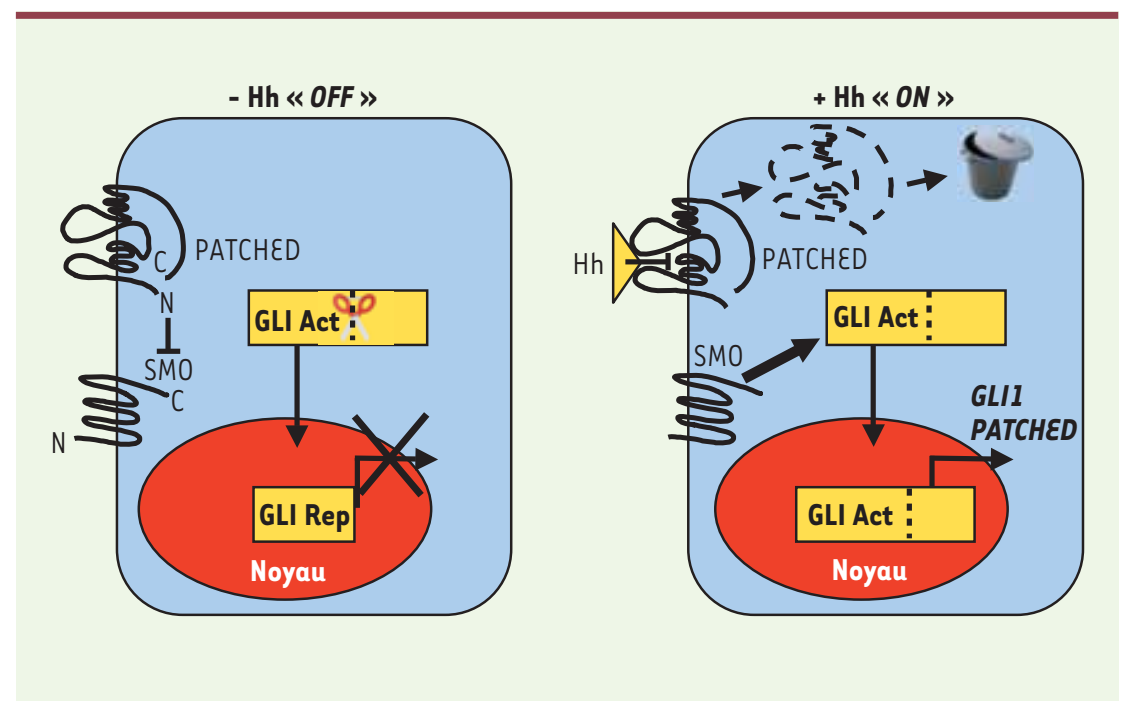

drome de Gorlin, I'holoprosencéphalie (mutations dans $\mathrm{SHH}$ ), les syndromes de Greig et de Pallister-Hall ${ }^{2}$ (mutations du facteur de transcription GLI3), constituent une famille de maladies génétiques du développement appelées « hedgehogopathies».

\section{PATCHED est un gène} suppresseur de tumeurs

Dans les CBC de patients atteints de syndrome de Gorlin, la mutation germinale de PATCHED (présente dans toutes les cellules de l'organisme) est généralement associée à la perte du second allèle (perte d'hétérozygotie) par délétion d'une portion de la région chromosomique $9 \mathrm{q} .22$ résultant en un génotype nullizygote $\left(\mathrm{PATCHED}^{-/-}\right)$. Dans les CBC

${ }^{1}$ L'holoprosencéphalie (HPE) est une malformation cérébrale complexe résultant d'une division incomplète du prosencéphale, survenant entre le $18^{e}$ et le $28^{e}$ jour de vie embryonnaire et affectant à la fois le cerveau et la face. Les enfants ayant une HPE présentent de nombreux problèmes médicaux: retard de développement et difficultés d'alimentation, épilepsie, instabilité de la température, du rythme cardiaque et de la respiration, troubles endocriniens.

Le traitement est symptomatique et palliatif. (source: orphanet)

${ }^{2}$ Le syndrome de Greig (transmission autosomique dominante) associe un hypertélorisme, une macrocéphalie avec bosses frontales proéminentes et une polysyndactylie. D'autres signes moins fréquents de la maladie incluent des anomalies du système nerveux central (SNC), des hernies et des troubles cognitifs. Le syndrome de Pallister-Hall, décrit en 1980, associe principalement une polydactylie centrale ou post-axiale, des syndactylies et un hamartome hypothalamique. Des malformations viscérales sont fréquentes (source : orphanet) sporadiques, une «première » mutation somatique de PATCHED liée aux UV (généralement une mutation changeant un $($ pour un $T$ ) est aussi très généralement accompagnée de la perte du second allèle. Tous les CBC, sporadiques ou secondaires à un syndrome de Gorlin, montrent que la voie est anormalement activée puisque ses transcrits cibles GLII et PATCHED s'accumulent en grande quantité dans la tumeur [8]. Le contrôle harmonieux de la transduction du signal SHH est donc absolument essentiel à la protection des kératinocytes épidermiques contre le développement en $\mathrm{CBC}[15]$.

\section{L'hypothèse du rôle déterminant des fibroblastes du stroma dans le développement des $\mathrm{CBC}$} Compte tenu du rôle essentiel de l'exposition UV dans le développement du $C B C$ sporadique, comment expliquer le développement de $C B C$ dans des zones cutanées qui ne sont pas exposées aux rayons UV chez les patients atteints de syndrome de Gorlin? Nous avons tenu compte de plusieurs éléments tirés des études du CBC sporadique : (1) le CBC est très rarement métastatique et l'invasion est locale; (2) ex vivo, contrairement aux cellules de carcinomes spinocellulaires (SCC; une tumeur épidermique également d'origine kératinocytaire mais

Figure 1. Représentation schématique de la transduction du signal Hedgehog (Hh). En l'absence du ligand Hh, PATCHED exerce une inhibition constitutive de l'activation de la voie. Les facteurs de transcription GLI2 et 3 (GLI act) subissent un clivage protéolytique (ciseaux) qui génère une forme de répresseur (GLI Rep) qui migre dans le noyau et inhibe la transcription des gènes cibles de la voie. En présence du signal Hh, PATCHED est internalisée puis dégradée par la voie des lysosomes. La dérépression de SMOOTHENED (SMO) conduit à la production des formes activatrices de GLI2 et 3 dont la translocation nucléaire aboutit à l'activation de la transcription des gènes cibles de la voie, notamment GLII et PATCHED.

susceptible de métastaser), les cellules de CBC ne se cultivent pas; (3) dès 1961 , des expériences de transplantation de $C B C$ in vivo révélaient le rôle essentiel du stroma péritumoral, essentiellement constitué de fibroblastes, pour la survie et la croissance des cellules épithéliales de la tumeur [9]. L'ensemble de ces observations suggérait que le $C B C$ est une tumeur peu plastique et très dépendante de son environnement stromal.

Nous avons donc exploré l'hypothèse selon laquelle les fibroblastes, population cellulaire majoritaire du stroma, pourraient jouer un rôle déterminant dans le développement des $C B C$, en particulier en territoire cutané non photoexposé. Grâce à des techniques de génie tissulaire, nous avons réalisé des cultures organotypiques de «derme Gorlin» - ces conditions de culture ont pour but de reproduire l'architecture tridimensionnelle du derme - à partir desquelles nous avons analysé l'expression du génome des fibroblastes sains (provenant de zones cutanées non photo-exposées) chez six patients différents ayant un syndrome de Gorlin. Nous avons comparé le «transcriptome Gorlin » au transcriptome de trois souches de fibroblastes primaires témoins obtenues à partir de sujets sains et cultivées dans les mêmes conditions. Les analyses ont révélé un répertoire 


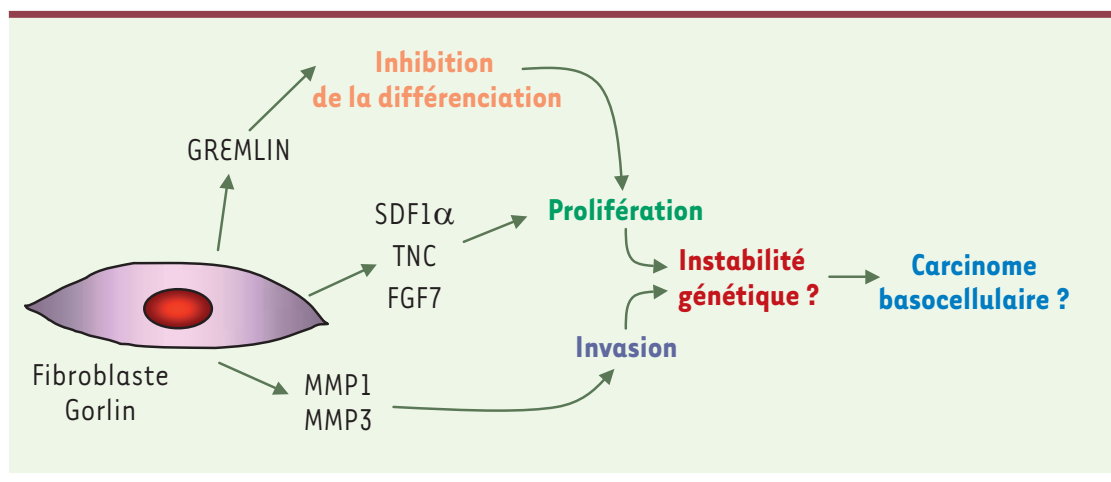

de 18 gènes dont les produits peuvent être impliqués dans le remaniement de la matrice extracellulaire, la régulation de la prolifération et de la différenciation kératinocytaires ou encore l'angiogenèse. De façon très surprenante, le répertoire des gènes exprimés de façon différentielle entre fibroblastes Gorlin et fibroblastes normaux (contrôles) est très similaire à celui des fibroblastes associés aux carcinomes sporadiques (carcinoma associated fibroblasts, (AF), déterminé après microdissection des tumeurs in vivo [10]. Ainsi, les ARN messagers des métalloprotéases matricielles MMP 1 (collagénase I), et MMP3 (stromélysine) s'accumulent fortement ( $\times 8,4$ et $\times 26,8$ respectivement) dans les fibroblastes de patients Gorlin par rapport aux fibroblastes contrôles. L'augmentation de la quantité des protéines correspondantes a été vérifiée. La surexpression de ces MMP, associée à la diminution de certains composants de la jonction dermoépidermique (collagène $7 \mathrm{Al}$, laminine A2) pourrait faciliter l'invasion du derme par des kératinocytes en dégradant la matrice extracellulaire et en fragilisant la membrane basale. Nos résultats ont aussi indiqué que les fibroblastes de patients Gorlin surexpriment les gènes CXCL12 (chemokine C-X-C motif, ligand 12) aussi nommé stromal derived factor (SDF1), FGF7 (keratinocyte growth factor) et ténascine C (TNC). De façon intéressante, la ténascine $C$ est un substrat des MMPl et 3 . La ténascine $C$ contient des motifs de type epidermal growth factor (EGF) libérés après clivage par ces MMP, et qui activent les récepteurs à l'EGF. Les produits de ces gènes sont connus pour stimuler la prolifération des kératinocytes, ce qui pourrait, dans un contexte matriciel favorable, contribuer à l'envahissement kératinocytaire du derme. Finalement, dans la mesure où prolifération et différenciation sont des processus mutuellement exclusifs dans les kératinocytes épidermiques, la surexpression de GREMLIN 1, un inhibiteur de la voie des bone morphogenic proteins (BMP, qui ont une activité qui favorise la différenciation), favorise probablement aussi le caractère «peu différencié/fortement prolifératif » des cellules tumorales du CBC. Dans leur ensemble, ces résultats indiquent qu'en I'absence de stress exogène la mutation d'un allèle de PATCHED dans les fibroblastes de patients suffit à leur conférer un phénotype péjoratif, très probablement impliqué dans la prolifération des kératinocytes épidermiques et l'invasion du derme [11]. Parallèlement, les données récentes obtenues par l'équipe de Frédéric de Sauvage ont montré que dans un grand nombre de tumeurs épithéliales l'activation de la voie SHH/ PATCHED prend place dans le stroma en réponse aux cellules tumorales, ce tés invasives [12]. Ces données confirment l'importance du rôle que pourraient jouer les fibroblastes de patients Gorlin dans le développement des CBC. D'une façon générale, le stroma apparaît donc aujourd'hui comme une nouvelle cible de traitements antitumoraux. La découverte d'une signature péjorative dans les fibroblastes de patients Gorlin, exprimée qui pourrait promouvoir leurs capaci-
Figure 2. Représentation synthétique des effets probables de l'altération du transcriptome dans les fibroblastes de patients Gorlin. MMP : metalloprotéases; TNC: ténascine; SDFl (ou CXCL12): stromal derived growth factor 1 ; FGF7 (keratinocyte growth factor).

spontanément et sans stress génotoxique évident, suggère que la contribution du stroma est particulièrement importante dans ce syndrome. De plus, d'autres travaux du laboratoire ont révélé les capacités invasives des kératinocytes de patients Gorlin dans les cultures de peau organotypiques ex vivo comportant un épiderme Gorlin et un derme composé de fibroblastes témoins [13]. Tous ces résultats suscitent de nouvelles interrogations. Chez les patients atteints de $C B C$ dans le contexte d'un syndrome de Gorlin, l'association des altérations des fibroblastes décrites ici à celles des kératinocytes est-elle suffisante pour générer une instabilité génétique puis un $C B C$ en l'absence d'exposition aux UV (Figure 2) ? Sur le plan thérapeutique, l'importance du derme est-elle suffisamment grande pour qu'une pharmacologie capable de normaliser le transcriptome des fibroblastes suffise à limiter le développement des CBC chez ces patients? $\diamond$ Dermal fibroblasts exert a key influence in the development of skin cancers: the model of Gorlin syndrome

\section{REMERCIEMENTS}

Les auteurs remercient chaleureusement $M^{m e}$ Chevallier-Lagente, les docteurs Philippe Dessen, Vladimir Lazar, Stéphanie Barnay-Verdier, Thomas Robert, Hugue Ripoche, Florence Brellier, et l'Institut Gustave Roussy pour leur contribution et leur soutien.

La recherche a été soutenue par l'Institut national du cancer (INCa), l'Association pour la recherche sur le cancer, la Fondation de l'Avenir, la Société française de dermatologie, à qui nous adressons également nos plus sincères remerciements. 


\section{CONFLIT D'INTÉRÊTS}

Les auteurs déclarent n'avoir aucun conflit d'intérêts concernant les données publiées dans cet article.

\section{RÉFÉRENCES}

1. Kalluri R, Zeisberg M. Fibroblasts in cancer. Nat Rev Cancer $2006 ; 6$ : 392-401.

2. Hoeijmakers JH. Genome maintenance mechanisms for preventing cancer. Nature $2001 ; 411$ : 366-74.

3. Epstein $\varepsilon H$. Basal cell carcinomas: attack of the hedgehog. Nat Rev Cancer 2008 ; 8 : 743-54.

4. Gorlin RJ. Nevoid basal cell carcinoma (Gorlin) syndrome. Genet Med $2004 ; 6: 530-9$.

5. Goldstein AM, Bale SJ, Peck GL, DiGiovanna JJ. Sun exposure and basal cell carcinomas in the nevoid basal cell carcinoma syndrome. J Am Acad Dermatol $1993 ; 29: 34-41$.
6. Gailani MR, Stahle-Backdahl M, Leffell DJ, et al. The role of the human homologue of Drosophila patched in sporadic basal cell carcinomas. Nat Genet 1996 ; 14: 78-81.

7. Johnson RL, Rothman AL, Xie J, et al. Human homolog of patched, a candidate gene for the basal cell nevus syndrome. Science $1996 ; 272$ : 1668-71.

8. Unden AB, Zaphiropoulos PG, Bruce K, Toftgard R, Stahle-Backdahl M. Human patched (PTCH) mRNA is overexpressed consistently in tumor cells of both familial and sporadic basal cell carcinoma. Cancer Res $1997 ; 57: 2336-40$.

9. Van Scott $\varepsilon$ J, Reinertson RP. The modulating influence of stromal environment on epithelial cells studied in human autotransplants. I Invest Dermatol 1961; $36: 109-31$.

10. Sneddon JB, Zhen HH, Montgomery K, et al. Bone morphogenetic protein antagonist gremlin $l$ is widely expressed by cancer-associated stromal cells and can promote tumor cell proliferation. Proc Natl Acad Si USA 2006 ; 103 : 14842-7.

11. Valin A, Barnay-Verdier S, Robert T, et al. $\mathrm{PTCH}^{+/-}$ dermal fibroblasts isolated from healthy skin of Gorlin syndrome patients exhibit features of carcinoma associated fibroblasts. PLOS ONE 2009; 4 : e4818.

12. Yauch RL, Gould SE, Scales SJ, et al. A paracrine requirement for hedgehog signalling in cancer. Nature 2008 ; 455 : 406-10.

13. Brellier F, Bergoglio V, Valin A, et al. Heterozygous mutations in the tumor suppressor gene PATCHED provoke basal cell carcinoma-like features in human organotypic skin cultures. Oncogene 2008 ; 27 : 6601-6.

14. Delmas V, Larue L. How keratinocytes instruct melanocytes to deliver the pigment. Med Sci (Paris) $2008 ; 24: 147-8$.

15. Basset-Seguin N, Soufir N. Patched/Sonic Hedgehog pathway and basal cell carcinoma. Med Sci (Paris) $2004 ; 20: 899-903$.

\section{NOUVELLE}

\section{Nétrine et cellules souches, attirance ou répulsion?}

Audrey Petit

$>$ On dénombrerait à l'échelle de la planète quelque 2,5 millions de personnes atteintes d'une lésion handicapante de la moelle épinière ( $M E$ ) causée par un accident. Chaque année, on compte plus de 130000 nouvelles victimes, principalement de jeunes adultes. Les handicaps fonctionnels varient selon le niveau de la blessure. Malgré des progrès importants dans la compréhension des mécanismes de ces lésions, celles-ci demeurent permanentes, offrant très peu d'espoir de réhabilitation [1].

Un traumatisme de la $M E$ produit un kyste, c'est-à-dire une zone nécrosée remplie de liquide, isolée du tissu sain par une cicatrice gliale, barrière physique et moléculaire formée par les astrocytes réactifs qui s'opposent à la régénération axonale et au remplacement des cellules neurales perdues [2]. Les recherches actuelles visent à neutraliser les actions inhibitrices de la cicatrice gliale et à combler la zone cystique par la création d'un substrat permissif [3].
Cellules souches neurales

dans le système nerveux central et la moelle épinière

La découverte de l'existence de cellules souches neurales (CSN) dans la ME des mammifères adultes, y compris l'espèce humaine, a suscité de nouvelles perspectives thérapeutiques $[4,5]$. Les CSN, douées d'autorenouvellement, peuvent, in vitro, donner naissance aux trois principaux types cellulaires composant le système nerveux central (SNC) : les neurones et les cellules gliales, astrocytes et oligodendrocytes (Figure IA). Dans la $M E$, ces CSN sont localisées autour du canal épendymaire et elles se différencient exclusivement en cellules gliales. Les études effectuées chez l'animal démontrent que les CSN ont la faculté de se reproduire à l'épicentre d'une hémisection de la $M E$, mais ne se fixent pas dans cette zone inhospitalière et vont plutôt s'en éloigner [6], obstacle de taille à l'usage de ces cellules en médecine régénérative.

Chez les rongeurs adultes, ces cellules ont une migration très limitée in vivo.
University of Washington, Department of neurosurgery, 325,9 th avenue, Box 359655 Seattle, WA 98104, États-Unis. petit.audrey@gmail.com

Dans le système nerveux central, seules deux voies de migration ont été définies: les cellules progénitrices nées des CSN de la zone sous-ventriculaire migrent suivant le courant de migration rostrale, route unidirectionnelle menant aux bulbes olfactifs, et celles nées de la zone sous-granulaire migrent sur une courte distance dans la couche de cellules granulaires du gyrus dentelé [7]. Les mécanismes et les signaux qui orientent la migration des CSN adultes sont peu connus. Toutefois, lors du développement du SN, la migration des neuroblastes est régulée par l'intégration spatiotemporelle de signaux moléculaires attractifs ou répulsifs, dont les protéines des familles nétrines, slit, éphrines et sémaphorines [8, 9]. Or ces molécules continuent d'être exprimées chez l'adulte.

\section{Guidage et migration des cellules} souches neurales de la moelle épinière Dans notre laboratoire, nous avons cherché à analyser la réponse des CSN de la $M E$ adulte à ces signaux de guidage 